\title{
Understanding contraindications for transoral robotic surgery (TORS) for oropharyngeal cancer
}

\author{
Gregory S. Weinstein • Bert W. O’Malley Jr. • \\ Alessandra Rinaldo • Carl E. Silver • \\ Jochen A. Werner • Alfio Ferlito
}

Received: 10 October 2014 / Accepted: 13 October 2014/Published online: 20 October 2014

(C) Springer-Verlag Berlin Heidelberg 2014

Transoral robotic surgery (TORS) was invented at the University of Pennsylvania in 2004 and arose from a specifically designed translational research program which included airway mannequin, cadaver, and animal model experimentation. The success of these preclinical efforts led to the first prospective human clinical research trial of TORS in 2005 [1, 2]. In 2009 the outcomes report from a multi-institutional retrospective trial, led by Weinstein and O'Malley [3] at the University of Pennsylvania, was utilized by the United States Food and Drug Administration (FDA) to approve the use of the da Vinci Surgical System

This paper was written by members of the International Head and Neck Scientific Group (http://www.IHNSG.com).

G. S. Weinstein · B. W. O’Malley Jr.

Department of Otorhinolaryngology-Head and Neck Surgery, The University of Pennsylvania Health System, Philadelphia, PA, USA

A. Rinaldo · A. Ferlito $(\bowtie)$

University of Udine School of Medicine, Piazzale S. Maria della

Misericordia, 33100 Udine, Italy

e-mail: a.ferlito@uniud.it

C. E. Silver

Department of Surgery, Albert Einstein College of Medicine, Montefiore Medical Center, Bronx, NY, USA

C. E. Silver

Department of Otolaryngology-Head and Neck Surgery,

Albert Einstein College of Medicine, Montefiore Medical

Center, Bronx, NY, USA

J. A. Werner

Department of Otolaryngology-Head and Neck Surgery,

Philipp University, Marburg, Germany
(Intuitive Surgical Inc, Sunnyvale, CA, USA) for TORS in patients. TORS procedures have been described to manage pathologies at numerous anatomic sites from the glottis and hypopharynx to the nasopharynx and skull base [4-6]. The most commonly reported use of TORS for malignant disease, however, has been for oropharyngeal cancer, particularly tongue base or tonsillar cancer [3, 7, 8]. This paper presents the contraindications for TORS oropharyngeal cancer resection as developed and performed at the University of Pennsylvania.

One of the unique aspects of the adoption of TORS has been that the inventors of the techniques have remained the primary post-graduate teachers of the procedure worldwide. Two of the authors of this paper (GSW and BWO) developed a structured TORS training program and a Robotics Training Lab at the University of Pennsylvania at which over 300 surgeons have been trained to date. A major focus of the dissemination of TORS and of the training program has been the emphasis on the indications and contraindications of this new category of surgical procedures. Our goal in developing TORS contraindications has been to (1) minimize the vascular complications encountered during and after the procedures (2) ensure successful and reproducible functional outcomes and (3) to maximize the achievement of negative margins thus avoiding the additional morbidity of high intensity adjuvant therapy. The following is a categorized list of these contraindications:

Vascular contraindications to TORS for oropharyngeal cancer

1. Tonsillar cancer with a retropharyngeal carotid artery.

2. Epicenter of the tumor is in the midline of tongue base or valleculae which would put both lingual arteries at risk. 
3. Tumor adjacent to carotid bulb or internal carotid artery which will result in intraoperative exposure of the vessel.

4. Encasement of the carotid artery by the primary tumor (T4b) or by a metastatic neck node.

Functional contraindications to TORS for oropharyngeal cancer

1. Tumor resection requiring more than $50 \%$ of the deep tongue base musculature.

2. Tumor resection requiring more than $50 \%$ of the posterior pharyngeal wall.

3. Tumor resection requiring up to $50 \%$ of the tongue base as well as the entire epiglottis.

Oncologic contraindications to TORS for oropharyngeal cancer

1. All T4b cancers.

2. Posterolateral fixation of tonsillar cancers to the prevertebral fascia. This is assessed by utilizing bimanual palpation with one finger placed intraorally and the opposite hand palpating extraorally. Fixation can be assessed by gently rocking the soft tissue of the tonsillar fossa medially and laterally.

3. Unresectable neck disease.

4. Neoplastic related trismus.

5. Multiple distant metastases.

Non-oncologic contraindications to TORS for oropharyngeal cancer

1. A medical condition that precludes stopping antiplatelet medications or anticoagulants.

2. As with all surgical approaches, any systemic or degenerative disease which is associated with unacceptable morbidity or mortality during general anesthesia or during the postoperative period.

3. Non-cancer related trismus which prevents robotic access via the oral cavity.

4. Cervical spine disease that interferes with necessary patient positioning during TORS.

In summary, contraindications to TORS can be categorized into vascular, functional, oncologic and non- oncologic categories. Although recognition of contraindications is of particular importance for the novice TORS surgeon or those early in the learning process (defined as fewer than 20 TORS procedures performed), experienced surgeons should also consider the above recommendations to ensure the most favorable outcomes for their patients.

The overall goal of defining the contraindications as described above was to promote standardized en-bloc resections that are reproducible and teachable to experienced head and neck surgeons and as well as trainees. The specific contraindications were derived from an ongoing experience with over 1,400 patients to date who have undergone TORS resections at the University of Pennsylvania. The authors assert that TORS was not designed to "push the envelope" for the "superspecialist" but rather to provide a patient care approach and technical platform that is useful for all head and neck surgeons.

Conflict of interest The authors have no conflicts of interest or financial ties to disclose.

\section{References}

1. O'Malley BW Jr, Weinstein GS, Snyder W, Hockstein NG (2006) Transoral robotic surgery (TORS) for base of tongue neoplasms. Laryngoscope 116:1465-1472

2. Weinstein GS, O’Malley BW Jr, Hockstein NG (2005) Transoral robotic surgery: supraglottic laryngectomy in a canine model. Laryngoscope 115:1315-1319

3. Weinstein GS, O'Malley BW Jr, Magnuson JS et al (2012) Transoral robotic surgery: a multicenter study to assess feasibility, safety, and surgical margins. Laryngoscope 122:1701-1707

4. Wei WI, Ho WK (2010) Transoral robotic resection of recurrent nasopharyngeal carcinoma. Laryngoscope 120:2011-2014

5. O'Malley BW Jr, Quon H, Leonhardt FD, Chalian AA, Weinstein GS (2010) Transoral robotic surgery for parapharyngeal space tumors. ORL J Otorhinolaryngol Relat Spec 72:332-336

6. Ozer E, Alvarez B, Kakarala K, Durmus K, Teknos TN, Carrau RL (2013) Clinical outcomes of transoral robotic supraglottic laryngectomy. Head Neck 35:1158-1161

7. Park YM, Lee JG, Lee WS, Choi EC, Chung SM, Kim SH (2009) Feasibility of transoral lateral oropharyngectomy using a robotic surgical system for tonsillar cancer. Oral Oncol 45:e62-e66

8. Weinstein GS, O'Malley BW Jr, Cohen MA, Quon H (2010) Transoral robotic surgery for advanced oropharyngeal carcinoma. Arch Otolaryngol Head Neck Surg 136:1079-1085 